\title{
¿CUÁL ES EL CAMINO PARA LA RECONSTRUCCIÓN DE NOSOTROS MISMOS? CON OCASIÓN DE LA V OLIMPIADA LATINOAMERICANA DE FILOSOFÍA 2014
}

Mario Mejía Huamán

Universidad Ricardo Palma

\section{RESUMEN}

El presente artículo gira en torno a la convocatoria de la V Olimpiada Latinoamericana de Filosofía 2014, cuyo tema de reflexión es: ¿Cuál es el camino para la reconstrucción de nosotros mismos? Continuando con el planteamiento tradicional partimos del Principio de Identidad de Parménides que se formula como $p \rightarrow p$ o $p \leftrightarrow p$, y el principio de no contradicción como $\sim(p \& \sim p)$, pero que en las disciplinas sociales, como es el problema de la identidad nacional, no podemos ser nosotros mismos, si no somos capaces de diferenciarnos de los otros. Sólo encontrándonos a nosotros mismos o reconstruyendo nuestra identidad podremos reafirmarnos como personas y proyectarnos positivamente al futuro; pero, no para aislarnos de los demás sino para tratarnos con respeto e integrarnos al grupo mayor bajo el principio de unidad en la diversidad.

PALABRAS CLAVE

Olimpiadas / Identidad / Alteridad / Reconstrucción / Andino.

\begin{abstract}
This article has been located within the field of the recently-called-on " 5 th LatinAmerican Philosophy Olympiad, 2014", whose reflection topic is What is the right way to reconstruct ourselves as human beings? To continue the traditional proposal, we begin with Parmenides's Principle of Identity, formulated as $p \rightarrow$ $p$ or $p \leftrightarrow p$ and the non-contradiction principle such as $-(\mathrm{p} \&-\mathrm{p})$. But in social sciences, as it is the case of national identity, we cannot define our identity if we are unable to establish our differences with other people. It is only by discovering ourselves or reconstructing our identity that we will be able to get reaffirmed as human beings and project ourselves positively into the future; not with the purpose of isolating ourselves, but with the due respect to one another, to become integrated to the bigger group within the principle of unity in diversity.
\end{abstract}

\section{KEY WORDS}

Olympiads / Identity / Alterity / Reconstruction / Andean. 


\section{INTRODUCCIÓN}

En los cinco últimos años, en Latinoamérica se han venido celebrando olimpiadas en, Uruguay, Brasil, Argentina, Paraguay, Chile, Colombia y últimamente el Perú. Las olimpiadas se realizan en varias fases, primero, se realizan en los colegios primarios y secundarios de las ciudades participantes, luego los mejores expositores asisten a las olimpiadas nacionales y finalmente a las Olimpiadas Latinoamericanas.

En Lima, en el la Universidad Ricardo Palma, el año 2013, hemos tenido una Olimpiada Filosófica Universitaria con éxito; el presente año lo realizaremos en el II semestre del año en curso. El tema del 2012 fue ¿Cuál es el costo social del progreso?, del 2013 fue ¿Qué conocimiento es posible en una época de incertidumbre? Del 2014 es: ¿Cuál es el camino para la reconstrucción de nosotros mismos?

En cierta ocasión a nuestro amigo y maestro Mario Bunge le escribieron preguntando, si los países "Tercer Mundistas” debían cultivar primero, las ciencias aplicadas o las ciencias básicas. Desde luego la respuesta que se pensaba que daría el epistemólogo era "las ciencias aplicadas", sin embargo el filósofo respondió en el sentido de que debíamos cultivar las ciencias básicas, esto es las ciencias teóricas, porque estas, son los que van a servir de base a las ciencias aplicadas.

El filósofo no está refiriéndose que estudiemos primero filosofía occidental, historia universal, Ciencias Naturales en abstracto sino, estudiar nuestra concepción del mundo y si tenemos una filosofía propia, primero lo nuestro; una historia para conocer cómo llegaron nuestros antecesores, a este país, ¿Qué hicieron para sobreponerse a la hostilidad del medio ambiente andino? ¿Qué bienes materiales y espirituales crearon para llegar al Estado Colectivista Andino?

Pues, mientras no conozcamos nuestra realidad, no sabremos aprovechar lo nuestro, y como en la actualidad seremos países dependientes en todo orden de cosas, pero siempre proveedores de materias primas a los países capitalistas. Ahora bien la pregunta es:

\section{LA IDENTIDAD Y LA ALTERIDAD, CATEGORIAS PARA LA FORMACION DE UNA IDENTIDAD NACIONAL}

En cierta ocasión un pastor evangélico le había preguntado a un rabino, cómo el pueblo judío a pesar de estar diseminado por todo el mundo, 
desde la diáspora, se había considerado como nación. El intérprete de la Palabra le había respondido: 1. Porque los padres enseñan a sus hijos quiénes son. 2. Porque les enseñan la historia de su pueblo y 3 . Porque también les enseñan quienes son sus amigos y quieres sus enemigos.

Concebiríamos a partir del ejemplo que no es posible concebirse la identidad sin tomar en cuenta la categoría filosófica de alteridad: el uno y el otro.

Expresado en una teoría de conjuntos tendríamos lo siguiente:

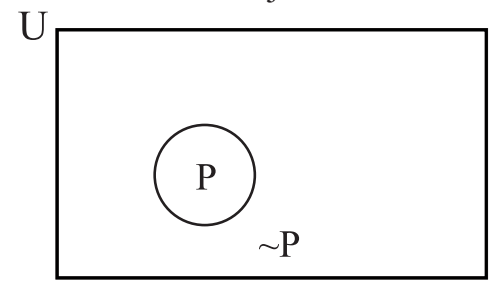

En el que:

1. El universo $U$ está formado por los elementos $P$ y $\sim P$

2. El elemento $P$ está definido por la circunferencia.

3. El espacio que se encuentra fuera de la circunferencia pertenece $\mathrm{a} \sim P$

Si el diagrama $P$ representara al universo de incas, el espacio restante correspondería a los no incas. Cualquier elemento que no es o pertenece a $P$ estaría fuera de la circunferencia.

Hemos recurrido a la representación lógica, toda vez que algunos expositores recurren al Principio de Identidad de Parménides y al Ser y Noser de Platón en el Parménides. En el que, el Ser es idéntico a sí mismo; Principio de Identidad. $P \rightarrow P, P \leftrightarrow P$; Principio de no-contradicción: $\sim(P$. $\sim P$ ); Principio de tercio excluido: $P v \sim P$ esto es, $P \rightarrow P$ o, en otras palabras, no es posible que $P$ sea $\sim P$, al mismo tiempo. Esto es: $\sim(P . \sim P)$. Entre estos autores que recurren a dicha expresión lógica podemos citar a María Luisa Rivara de Tuesta, Dora Vidal y Francisco Miró Quesada.

\section{POSICIÓN DE MARÍA LUISA RIVARA DE TUESTA}

Respecto a la identidad María Luisa Rivara de Tuesta sostiene: "cuando hablamos de identidad nacional no se está hablando de identidad 
de... cada grupo, por el hecho de estar ligados por elementos espaciotemporales y culturales pueden sentirse como que son miembros de un solo grupo y una sola cultura, con elementos propios y con un destino común".

Examinando como un objeto real 'lo nacional' y reduciendo este objeto real a lo idéntico, examinando en su historicidad y con sentido analítico para lograr su síntesis identificadora, se encuentra que la identidad nacional se constituye en una triple manifestación...Es necesario señalar que el pluriculturalismo, el plurietnicismo y el plurilinguismo constituyen una herencia ancestral en el Perú. (Rivara, La Identidad Nacional, 2001, p. 432)

Rivara de Tuesta sostiene a partir del mito de las dos creaciones de Juan Betanzos que el Perú es una nación pluricultural, pluriétnico y plurilingüe.

Lo que nos interesa poner de manifiesto del contenido de este mito de creación es su aspecto multiétnico, pluricultural y multiespacial. No se trata de una sola pareja de la cual ha de descender la humanidad, ni se trata de situar a esa única pareja en un paraíso; todo lo contrario: grupos humanos diversos, con localizaciones de origen diferente, lenguas distintas y usos y derecho (reglas morales) propios son los que constituyen la humanidad. (Rivara, 2001, p. 434)

\section{VÍCTOR ANDRÉS BELAUNDE}

El filósofo Víctor Andrés Belaunde sostiene que el peruano sufre de anatopismo, esto es, de alienación o enajenación: que nuestra identidad aún no está formada, que para ello debemos girar en torno a lo espiritual y religioso. Distingue cuatro tendencias al respecto:

- Peninsularista,

- Autoctonista,

- Fusionista y

La posición de peruanidad integral (Síntesis viviente). El filósofo escribe:

La teoría de la síntesis viviente, explica los fenómenos de transculturación. La historia de la cultura y de la formación nacional nos muestra el proceso en que los valores superiores de una cultura, al asumir los elementos de nuestras tierras, determinan nuevos matices culturales por la influencia en que se plasman, creando diversos espíritus nacionales.... (Belaunde, Evolución cultural de la época hispánica, 1987, Tom. V, cap. VII, p. 229) 
Las ideas del autor de Peruanidad sin duda corresponden al pensamiento católico y europeo del siglo pasado. Primero, cuando considera que "hay valores que son cohesionados, transformados, por los valores superiores" y cuando sostiene que "la cultura primitiva no desaparece del todo; lo que tiene de bueno o permanente es asimilado o eliminado". Consideramos que los términos "superiores" y "primitivo" a nuestra manera de ver, lindan con la apreciación despectiva de nuestra cultura prehispánica.

Para nosotros sería difícil admitir que la cultura traída por los conquistadores fue superior a la andina, a menos que esta sea tomada como primitiva. Pensamos que los prejuicios católicos aún fuertes hasta los setentas, fueron un impedimento para que el autor apreciara cabalmente a las culturas prehispánicas, por otro lado, todavía no se habían hecho estudios de la Visión Andina del Mundo y señalado las limitaciones de la visión occidental traído por los conquistadores, y que en el futuro no se constituirían en los cimientos de la pretendida cultura nacional unidimensional en actual formación.

Cuando íbamos a publicar en El Dominical del diario El Comercio de Lima, en la columna bilingüe Integración, un artículo sobre La esencia del hombre andino y el trabajo, que son completamente opuestas en ambas concepciones del mundo, Francisco Miró Quesada Cantuarias, dueño y director de El Dominical, con cierto temor nos dijo, Mira, Mario es posible que la Iglesia nos proteste por esto. Publicamos el artículo, causó cierta preocupación pero, como la diferencia de las visiones del mundo eran objetivamente diferentes, el público no tuvo sino que aceptar. Sólo tuvimos una protesta nuestra amiga, la rectora de la Universidad Femenina del Sagrado Corazón de entonces; ella nos dijo que estaba chocando con la Iglesia y que ello probablemente me costaría la supresión de mi contrato. Le respondí en el sentido de que lo que escribía no era un invento mío, y que por ello podía poner mi cabeza en la guillotina. Felizmente no me despidieron del trabajo.

\section{ALBERTO WAGNER DE REYNA.} sostiene:

María Luisa Rivara cita al filósofo Alberto Wagner de Reyna, quien

Nuestro destino es elaborar sobre la base de la tradición ibérica una concepción del mundo y un tenor de vida dignos de sus fundamentos y a la vez nuevos, gracias a nuestra especial situación histórica, geográfica y étnica.” (Rivara, 2001, p. 440) 
A esa concepción del mundo fundada en la tradición ibérica de concesión del mundo Wagner de Reyna añade:

...la emoción por lo indígena y sus valores vitales, en la conciencia de la creación de una cultura original y de abolengo, a la vez vieja y nueva, de Europa y América, nuestra y del mundo (Rivara, 2001, p. 440).

Por ejemplo, respecto a nuestra inautenticidad filosófica sostiene que: “...el futuro filosófico de la cultura de Iberoamérica es continuar el legado helénico-cristiano que se impuso frente al aborigen de América, pero no simplemente como imitadores y divulgadores de los grandes temas del filosofar occidental, sino como creadores, a través de la reflexión filosófica de una cultura nueva y original (que incluya a la indígena) ..." "Y en esta gran obra... lo indígena será el nuevo soplo de viento en las velas..." (Rivara, 2001, p. 440), como en el viaje de Colón al Nuevo Mundo.

Finalmente, respecto a nuestra identidad, el autor afirma que, primero debemos develar nuestra identidad en nosotros mismos y reforzarla, luego, dar y recibir los valores positivos de ambas culturas. Hoy por hoy diríamos, primero debemos encontrar o reconstruir nuestra identidad auténtica, no la postiza.

\section{AUGUSTO SALAZAR BONDY}

Es otro autor pensador nacional en quien se pueden encontrar opiniones sobre la identidad y la autenticidad. En iExiste una filosofía de nuestra América?, sostiene que:

Los hispanoamericanos estamos claramente en el caso de este existir inauténtico... vivimos alienados respeto a la propia realidad a la integración y por ende sin vigor espiritual. (Salazar Bondy, 1968, pp. 81-88)

Salazar Bondy fue en los años sesenta el ideólogo de la "Reforma Educativa", del entonces denominado Gobierno Revolucionario escribió:

... es importante siempre pensar en lo que la educación va a ser y en lo que el cambio de conciencia va a ser, para liquidar las dominaciones. Por eso es importante que, en toda revolución, al hilo de la revolución social y económica, se dé siempre una revolución cultural. (Salazar Bondy, 1995, pp. 184) 
Sin embargo, Salazar Bondy no propuso las bases sobre los que se debe formar la identidad y la autenticidad nacional, sí sobre lo occidental o sobre lo andino.

\section{FRANCISCO MIRÓ QUESADA}

El filósofo peruano más conocido de todos los tiempos, toma la identidad en tres sentidos; En un primer sentido, se refiere a identificación, rasgos reconocibles y diferenciales de las cosas; en un segundo sentido, se refiere a una sociedad determinada, en que por lo menos una gran mayoría de los miembros de dicha sociedad, tienen rasgos comunes y tienen conciencia de tenerlo.

En un tercer sentido sostiene que la identidad es

... más bien un ideal, se refiere a los rasgos comunes que no tienen los miembros de una sociedad sino a los que nosotros quisiéramos que tuviera y agrega, no distinguir entre esta identidad ideal y la identidad real de nuestro país es fuente de muchas confusiones y de no pocos errores cuando se trata de juzgar lo que realmente somos y lo que podemos ser. (Rivara, 2001, p. 445)

Considera que son significativos los cambios que están ocurriendo en las principales ciudades del Perú, principalmente en la Costa peruana como Lima, donde la migración masiva de los 50 y 60, añadidas a las que fueron ocasionadas por el fenómeno del terrorismo, iniciaron significativos cambios. En tal sentido escribe:

... en los últimos decenios, debido a que se está dando un fenómeno de migración andina, se está produciendo una simbiosis de formas occidentales modernas con formas autóctonas, lo que está creando una realidad social y cultural completamente nueva. Este cambio, de vastas dimensiones e incontenible, permitiría que el Perú, a pesar de su actual heterogeneidad, termine por integrarse cultural y socialmente.

Se trata de un desafío dirigido a forjar un nuevo tipo de sociedad en la que todo ciudadano, en la palpitante plenitud de la conciencia colectiva, sienta que ser peruano le confiere una existencia llena de riqueza humana. (Rivara, 2001, pág. 445)

Consideramos que como sostienen los filósofos debemos sentirnos que la peruanidad nos confiere una riqueza humana en nuestra existencia, 
pero esto es, a nivel de Estado, más a nivel de naciones no es posible ir contra la realidad, el Estado peruano está conformado por más de cien naciones. Lo que debemos hacer es buscar la unidad en la diversidad, que sin renunciar a la identidad real, por el momento velada, enajenada, alienada, podamos vivir respetándonos; respetando el uno al otro, esto es "allin kawsay", vivir bien; la pretendida tolerancia, concepto que viene a nosotros de los pensadores americanos, no es sino soportarnos hasta que en un momento dado no podemos más apretar el resorte.

En quechua al hecho de la alienación se denominaba como "ch'utikuy" esto es quitarse lo suyo y ponerse lo extraño, esto es convertirse en "otro", (hoq kay). El ch 'utikuy tenía como destino final el "mistiyay". El objetivo de la Olimpiada Filosófica del 2014 podríamos traducirlo al quechua como " $k i$ kin kayninchisman kutiykuy (el retorno a nuestra identidad), para vivir como somos en realidad: "kikin kayninchisman hina kawsananchispaq", para vivir "kikin kayninchismanta pacha". Esto es no para quedarnos retrasados en el espacio y tiempo, sino crecer a partir de nosotros mismos.

\section{DORA VIDAL}

En una ponencia sustentada en la Pontificia Universidad Católica sostiene que "El análisis sobre la identidad como lo "mismo"...se encuentra ya planteado desde Parménides ("Es lo mismo pensar y ser"). "Platón en el Parménides analiza que al considerarse que el Ser es Uno, podría decirse que es el "Mismo" siempre. ... no es posible ninguna otra manera de ser más allá del ser. De ello se sigue que para hablar del Ser como Uno y como lo "Mismo" es necesario considerarlo en relación con lo Otro.... Si el ser es Uno e inmóvil, el No-Ser vendría a ser movimiento, pluralidad".

A Dora Vidal le vi trabajando sobre el tema desde hace más de una década, pero dada las limitaciones que muchos profesores tenemos en un país subdesarrollado, ha tardado en hacer conocer sus investigaciones, autores de otros países que trabajaban en el mismo tema sacaron a luz sus investigaciones antes que ella. En su ponencia hace mención a Parménides, Platón y Heidegger, y respecto a la identidad humana dice:

La unificación de la existencia integral se manifiesta como "yo", "mi mismo" e incluye tanto sus manifestaciones materiales como espirituales, en el sentido de auto identificación o pertenencia.

Tal unidad se manifiesta como tensión dinámica...por lo menos dos sentidos: a) de afirmación respecto a aquella que le permite mante- 
nerse en la existencia, enriquecerse y desarrollar sus propias potencialidades (posibilidades), b) de negación, la identidad es a su vez excluyente de aquello que considera que no es sí misma,... por eso tienen que pugnar con estas otras fuerzas que al oponérsele, intentan invadirla o disolverla y anularla.

En el mundo contemporáneo multicultural las identidades pugnan por el reconocimiento que significa la exigencia del respeto a las diferencias por la igualdad dignidad de todas las identidades. Exigen por ello, el derecho a la palabra, al diálogo intercultural, más allá de la tolerancia, buscan ser reconocidas precisamente como diferentes. (Vidal, 2000, pp. 463-466)

\section{¿CUÁL ES EL CAMINO PARA LA RECONSTRUCCIÓN DE NOSO- TROS MISMOS?}

La pregunta implica conceptos como que nosotros, no somos "nosotros mismos", sino que estamos alienados, estamos enajenados. No queremos admitir nuestra identidad nacional, ni personal. En cierta ocasión la Dra. Gloria Benavides profesora de la Universidad Femenina del Sagrado Corazón, pidió en clase que las estudiantes provenientes de la sierra levantaran la mano para identificarse. Para su sorpresa sólo una estudiante y la profesora se identificaron como que procedían de la sierra. La estudiante procedía de la sierra de su país. Por lo general, nosotros mientras conozcamos nuestro pasado e identifiquemos lo valioso de nuestro territorio y mientras no nos identifiquemos con sus valores, tendremos temor de levantar la mano.

Por lo general quienes estamos orgullosos de ser indios como el que habla, serranos o andinos, somos los que hemos logrado alcanzar nuestra identidad nacional y personal, generalmente por esfuerzo propio o por la educación.

El historiador y filósofo, José Tamayo Herrera y el filósofo Francisco Miró Quesada Cantuarias son los que en mi caso hicieron que llegara al lugar que me encuentro en la filosofía nacional y latinoamericana. El primero, quien fue mi profesor en la Universidad San Antonio Abad del Cusco, en cierta ocasión me dijo, si quieres ser grande entre los filósofos occidentales, tendrías que dominar el alemán, inglés o francés. Quien conozca bien y refutar a Hegel, Kant o Heidegger, deberá ser un alemán. Tú podrías ser grande si haces filosofía de lo nuestro, a partir del quechua que dominas.

El filósofo Francisco Miró Quesada, me escuchó discutir en un congreso nacional de filosofía, no recuerdo qué tema, pero concluí la 
disputa manifestando que "en el pensamiento quechua la cosa no era asi". Desde esa ocasión fuimos grandes amigos y aprendimos el uno del otro. Él adquirió mejor información de la concepción andina del mundo y para mí conversar semanalmente con el sabio, por más de cinco años, fue un posgrado privado, con uno de los mejores filósofos en nivel mundial de la segunda mitad del Siglo XX.

Por supuesto, no todos deberán pasar por la misma experiencia que pasé pero el campo de la cultura andina y peruana es aún virgen, lo mismo que su espacio físico y sus riquezas.

a. Los Andes necesitan literatos y críticos de la literatura para mostrar nuestra sabiduría y arte oral. En la conquista y colonia han maquillado nuestra poesía por ejemplo, y nos han presentado con una máscara de la poesía occidental, habiendo quitado lo íntimo de la belleza de nuestra poesía. La métrica española es a la poesía quechua como unos zapatos de gringo para nuestros pies. Autores, como Szeminski, no han hecho sino malinterpretar nuestra concepción del mundo al no conocer el quechua. Los mismos podemos decir de otros críticos literarios como Luís Alberto Sánchez, para quien la palabra amor significa urpi, o para María Rostowrowski, para quién no existe en quechua la palabra Dios.

b. Fortunato L. Herrera, un profesor de biología de la Universidad del Cusco a mediados del Siglo pasado, sólo caminando de su casa a la universidad a pié, descubrió y clasificó más de un centenar de plantes desconocidas para la botánica. Su clasificación, en más del \% 75 está vigente. En la selva, cuantas plantas esperan tener con vuestros apellidos carta de ciudadanía.

c. Siendo nuestros pueblos andinos colectivistas tenemos que recibir el comunitarismo de Robert Bellah, Charles Taylor, Michel Walzer, Alasdair MacIntayre.

d. Como sostiene Gamaliel Churata (Arturo Peralta): "El mito griego es al alma máter del mundo occidental; el mito inkásico debe serlo de un América del Sur con ego". (Peralta, 1957, p. 33).

e. Nosotros tenemos entre las principales publicaciones de la Editorial Universitaria de la Universidad Ricardo Palma: Filosofia andina de la Naturaleza (2008). Teqse: la cosmovisión andina y las categorías quechua como fundamentos para una filosofía peruana y de América andina (2010). En quechua y español: Anti Yachay Wayllukuy (Filosofía Andina, 2011). Hacia una filosofía 
andina; Quechua; 40 lecciones, con más de 10,000 ejemplares vendidos por la Universidad. Diccionario Castellano-Quechua, Curso de Introducción a la Lógica. Introducción a la Historia de la Filosofia. Esperan su publicación: Curso de quechua para Ciencias de la Salud e Introducción a la filosofía andina.

f. Fuimos director la columna bilingüe Hukllanakuy - Integración en El Dominical de El Comercio de Lima, por más de cinco años. Exponiendo temas de Filosofía Andina, hemos asistido a tres congresos mundiales de Filosofía, dos de los cuales con compañía del sabio Miró Quesada, y a otros congresos internacionales de filosofía por América, Europa, países del norte, y en dos oportunidades a Moscú.

\section{IMPORTANCIA DEL QUECHUA EN LA CONSERVACIÓN DE LA IDENTIDAD NACIONAL ANDINA}

Para los andinos, el quechua es nuestro primer idioma, es un instrumento de comunicación íntima, es un elemento vivo de nuestra cultura nacional originaria que a pesar de los pronósticos de propios y extraños sigue viva y adquiere mayor fuerza, pues hoy, hay ciudadanos más comprometidos con su conservación, y su aprendizaje es formal como de cualquier otro idioma. Muchas universidades del Perú y del extranjero tienen entre su currículo, los idiomas andinos, el quechua en sus distintas variantes. Así tenemos en el extranjero se enseña quechua en varias universidades de Estados Unidos de Norteamérica, en la Sorbona de París, en Alemania, en Suecia, en Moscú, en Japón. En el Perú, hoy, es un idioma contado como requisito para los grados de magister y doctor.

El quechua como cualquier idioma vivo, se actualiza de acuerdo a su capacidad onomatopéyica y al recibir "préstamos" como lo hacen todos los idiomas. En los pueblos de la sierra, principalmente del sur oriente peruano y del noroeste boliviano, las propagandas comerciales se emiten en quechua y aimara, entre otros programas, los partidos de futbol son transmitidos en el idioma nativo. Los párrocos, pastores evangélicos y los israelitas utilizan el quechua en sus prédicas y estudios bíblicos. Como en todos los idiomas que han adquirido carta de ciudadanía, con la publicación de las obras cumbres de la literatura nacional y la Biblia, en el Perú, se han publicado biblias en el quechua inka, dos ediciones, en quechua ayacuchano, quechua de Huallaga, así como Nuevos Testamentos en otras tantas variantes, como en quechua de San Martín, del Callejón de Huaylas, y en el quechua de Apurímac. 
Para la publicación de la Biblia se han seguido los siguientes pasos previos, primer contacto con la cultura, inventario de términos, proceso de dotación de escritura y de gramática propia por lingüistas de alto nivel de preparación, al mismo tiempo de enseñanza y alfabetización por los profesores; traducción de pasajes bíblicos, vida de personajes bíblicos, Nuevos testamentos y finalmente después de unos 50 o más años borradores de las biblias, las mismas que una vez escrito los borradores, son traducidos al inglés, pasan luego a la revisión de especialistas en griego y hebreo, con cuyas observaciones, se redacta la versión final.

La educación debe consistir en la formación e información de la cultura en la lengua materna y de la lengua oficial del Estado. Esto para las poblaciones andinas quechua-hablantes. Para los ciudadanos castellano hablantes de las ciudades que le podríamos llamar metrópolis, debe ser requisito para el estudio de la historia ancestral del Perú, en un horario de 3 sesiones durante un año y en la universidad debe enseñarse como cualquier otro idioma en ciclos intensivos, esto es a dos horas diarias, durante un año, para los estudiantes cuyas carreras exigen el servicio civil del graduando y con aquellas en que la profesión exige el trato con personas que no hablan el castellano.

A modo de conclusión consideramos que uno de los caminos para encontrarnos a nosotros mismos y reconstruir nuestra identidad es el quechua, puesto que los idiomas son las claves o puertas para acceder cabalmente a las culturas. Por otro lado tenemos que tener la voluntad de reconstruirnos a nosotros mismos, sin esta voluntad, vano serán los esfuerzos para buscar los caminos. Necesitamos estar conscientes de la necesidad de reconstruirnos a nosotros mismos.

\section{REFERENCIAS BIBLIOGRÁFICAS}

BELAUNDE, Victor Andres

1987 Evolución cultural de la época hispánica. En V.A. Belaunde, Peruanidad (p. 229). Lima: Edición de la Comisión Nacional del Centenario. (Obras completas).

PERALTA, A.

1957 El Pez de Oro. La Paz: Canata.

RIVARA, María Luisa

2001 La identidad Nacional. En M. L. Rivara, Filosofia e historia de las ideas en el Perú (p. 445). Lima: Fondo de Cultura Económica. 
SALAZAR BONDY, Augusto

1968 ¿Existe una filosofia de nuestra América? México: Siglo XXI Editores. 1995 El pensamiento Latinoamericano en el contexto del tercer mundo. Dominación y liberación. Escritos 1966-1974. Lima: Fondo Editorial de la Facultad de Letras de la Universidad Nacional Mayor de San Marcos.

VIDAL, Dora

2000 El concepto de identidad humana y la posibilidad de su aplicación en el sistema educaional. En M.E. Giusti, La filosofía del siglo XX: Balance y perspectivas (pp. 463-466). Lima: Pontificia Universidad Católica del Perú, Fondo Editorial. 